\title{
On the exact solution of incompressible viscous flows with variable viscosity
}

\author{
A. Fatsis ${ }^{1}$, J. Statharas ${ }^{2}$, A. Panoutsopoulou ${ }^{3} \&$ N. Vlachakis ${ }^{1}$ \\ ${ }^{I}$ Technological University of Chalkis, Department of Mechanical \\ Engineering, Greece \\ ${ }^{2}$ Technological University of Chalkis, Department of Aeronautical \\ Engineering, Greece \\ ${ }^{3}$ Hellenic Defence Systems, Greece
}

\begin{abstract}
Flows of variable viscosity fluids have many industrial applications in fluid mechanics and in engineering such as pump flows for highly viscous fluids. Carrying out a literature survey, it was found that in most cases the fluid viscosity is mainly temperature dependent. Numerical investigation of such flows involves the solution of the Navier-Stokes equations with an extra difficulty arising from the fact that the viscosity is not constant over the flow field. This article presents an analytical solution to the Navier-Stokes equations for the case of laminar flows in rotating systems with variable viscosity fluids. The equations are written in a cylindrical relative frame of reference rotating with a constant angular velocity. In the following, appropriate reference quantities are chosen to provide the non-dimensional form of the partial differential equations. The proposed solution that satisfies the continuity, the momentum and the energy equation, is expressed in terms of the Bessel function of the first kind and of exponential functions. Carrying out algebraic manipulations, it is proven that the proposed solution satisfies all the governing equations. Plots of the velocity, pressure and temperature distributions show the influence of the radius and of the axial coordinate to the flow field.

Keywords: variable viscosity, exact solution, Navier-Stokes, incompressible flow, laminar flow, Bessel functions.
\end{abstract}




\section{Introduction}

Exact solutions of the Navier-Stokes equations are very important not only because they are solutions of certain fundamental flows but also because they serve as accuracy checks for experimental, numerical, empirical and asymptotic methods An excellent review summarizing the basic steady and unsteady flows providing their exact solutions is presented by Drazin and Riley [1].

These are exact solution methods, treating non-Newtonian fluids, such as polymer solutions, special soap solutions, blood, paints, certain oils and greases, for which a different model has to be used to account for the non-Newtonian behavior. Such a model based on the theory of Oldroyd [2], is described by Fetecau [3].

The exact solutions of the Navier-Stokes equations when the viscosity is variable are rare, however the literature in which the viscosity is variable is dependent upon the space, time, temperature, pressure etc. Martin [4] used for the first time an elegant method in the study of the Navier-Stokes equations for an incompressible fluid of variable viscosity. Martin reduced the order of the governing equations from second order to first order by introducing the vorticity function and the generalized energy function.

Naeem and Nadeem [5] generalized Martin's approach to study the steadystate, plane, variable viscosity, solving the incompressible Navier-Stokes equations. They transformed the equations to a new system with viscosity, vorticity, speed and energy function. The transformation matrices included the unknown functions and helped determine some exact solutions for vortex, radial and parallel flows.

Naeem [6] presented recently a class of exact solutions of the equations governing the steady plane flows of incompressible fluid of variable viscosity for an originally specified vorticity distribution.

The effects of linearly varying viscosity and thermal conductivity on steady free convective flow of a viscous incompressible fluid along an isothermal vertical plate in the presence of heat sink were investigated by Mahanti and Gaur [7]. The governing equations of continuity, momentum and energy are transformed into coupled and non-linear ordinary differential equations using similarity transformation and then solved using Runge-Kutta fourth order method.

Variable viscosity Couette flow was investigated by Makinde and Maserumule [8] by solving analytically the Navier-Stokes equations using a perturbation method coupled with a Hermite-Pade approximation technique to obtain the velocity and temperature distributions.

This article presents a method providing analytical solutions to the incompressible Navier-Stokes equations. The equations are written in cylindrical coordinates suited for applications in rotating machinery flows. It is proven that the analytical solutions obtained satisfy the partial differential equations. 


\section{Governing equations}

Considering that the model aims to describe the motion of a Newtonian fluid, the Navier-Stokes equations are the governing equations of the problem [9]. It was chosen to express the equations in cylindrical coordinates because it is more convenient for axisymmetric bodies or rotating systems. Moreover since many applications of rotating systems concern fluid rotating machinery such as compressors, turbines or pumps, the relative frame of reference is preferred. Adopting the relative cylindrical frame of reference, $(r, \theta, z)$, the $z$-axis coincides to the axis of rotation, the $r$-axis is directed radially outwards and the $\theta$-axis is in the peripheral direction and it is rotating with a constant rotational speed with the rotor. In this case the relative velocity is linked to the absolute velocity and the rotation speed of the relative system of coordinates:

$$
\vec{V}=\vec{W}+\vec{U}=\vec{W}+(\vec{\omega} \times \vec{r})
$$

where $\vec{V}=v_{r} \cdot \vec{i}_{r}+v_{\theta} \cdot \vec{i}_{\theta}+v_{z} \cdot \vec{i}_{z} \quad$ is the absolute velocity vector, $\vec{W}=u_{r} \cdot \vec{i}_{r}+u_{\theta} \cdot \vec{i}_{\theta}+u_{z} \cdot \vec{i}_{z}$ is the relative velocity vector and $\vec{U}=(\vec{\omega} \times \vec{r}) \cdot \vec{i}_{\theta}$ is the rotating speed of the relative system of coordinates.

The following equation was chosen to express the viscosity in terms of temperature, as proposed by Schilling et al. [10]:

$$
\mu=\mu_{0} \cdot(1-\beta \cdot T)
$$

where $\beta$ is the thermal expansion of the fluid.

The system of the 3-D Navier-Stokes equations for incompressible flow in cylindrical coordinates can be non-dimensionalised by using the following parameters:

$$
\left.\begin{array}{c}
u^{*}=\frac{u_{r}}{\omega \cdot R}, v^{*}=\frac{u_{\theta}}{\omega \cdot R}, w^{*}=\frac{u_{z}}{\omega \cdot R}, R^{*}=\frac{r}{R}, z^{*}=\frac{z}{R}, H^{*}=\frac{\mu}{\mu_{0}} \\
P^{*}=\frac{P}{\rho \cdot(R \cdot \omega)^{2}}, T^{*}=\frac{c_{p} \cdot T}{(R \cdot \omega)^{2}}, \operatorname{Re}=\frac{\rho \cdot R \cdot \omega^{2}}{\mu_{0}}, E c=\frac{\beta \cdot(R \cdot \omega)^{2}}{c_{p}}
\end{array}\right\}
$$

where $R$ is a characteristic radius of the geometry in consideration, $R e$ and $E c$ are the non-dimensional Reynolds and Eckert numbers based on the rotational speed and the radius of the rotor, [9].

Thus, the continuity equation can be written:

$$
\frac{\partial u^{*}}{\partial R^{*}}+\frac{u^{*}}{R^{*}}+\frac{\partial w^{*}}{\partial z^{*}}=0
$$


Using the above non-dimensional parameters, the $r$-momentum equation can be written:

$$
\begin{gathered}
u^{*} \cdot \frac{\partial u^{*}}{\partial R^{*}}+w^{*} \cdot \frac{\partial u^{*}}{\partial z^{*}}-\frac{v^{* 2}}{R^{*}}=-\frac{\partial P^{*}}{\partial R^{*}}+\frac{H^{*}}{\operatorname{Re}} \cdot\left(\frac{\partial^{2} u^{*}}{\partial R^{* 2}}+\frac{1}{R^{*}} \cdot \frac{\partial u^{*}}{\partial R^{*}}-\frac{u^{*}}{R^{*}}+\frac{\partial^{2} u^{*}}{\partial z^{* 2}}\right)+ \\
R^{*}+2 v^{*}+\frac{1}{\operatorname{Re}} \cdot \frac{\partial H^{*}}{\partial z^{*}} \cdot \frac{\partial u^{*}}{\partial z^{*}}+\frac{1}{\operatorname{Re}} \cdot \frac{\partial H^{*}}{\partial z^{*}} \cdot \frac{\partial w^{*}}{\partial R^{*}}+\frac{2}{\operatorname{Re}} \cdot \frac{\partial H^{*}}{\partial R^{*}} \cdot \frac{\partial u^{*}}{\partial R^{*}}
\end{gathered}
$$

The non-dimensional form of the $\theta$-momentum equation is:

$$
\begin{gathered}
u^{*} \cdot \frac{\partial v^{*}}{\partial R^{*}}+w^{*} \cdot \frac{\partial v^{*}}{\partial z^{*}}+\frac{u^{*} \cdot v^{*}}{R^{*}}= \\
-2 \cdot v^{*}+\frac{H^{*}}{\operatorname{Re}}\left[\frac{\partial}{\partial R^{*}}\left(\frac{\partial v^{*}}{\partial R^{*}}\right)+\frac{1}{R^{*}} \cdot \frac{\partial v^{*}}{\partial R^{*}}-\frac{v^{*}}{R^{* 2}}+\frac{\partial}{\partial z^{*}}\left(\frac{\partial v^{*}}{\partial z^{*}}\right)\right]+ \\
+\frac{1}{\operatorname{Re}} \cdot \frac{\partial \mathrm{H}^{*}}{\partial z^{*}} \cdot \frac{\partial v^{*}}{\partial z^{*}}+\frac{1}{\operatorname{Re}} \cdot \frac{\partial \mathrm{H}^{*}}{\partial R^{*}} \cdot\left(\frac{\partial v^{*}}{\partial R^{*}}-\frac{v^{*}}{R^{*}}\right)
\end{gathered}
$$

The non-dimensional form of the $z$-momentum equation is:

$$
\begin{gathered}
u^{*} \cdot \frac{\partial w^{*}}{\partial R^{*}}+w^{*} \cdot \frac{\partial w^{*}}{\partial z^{*}}=-\frac{\partial P^{*}}{\partial z^{*}}+\frac{H^{*}}{\operatorname{Re}} \cdot\left(\frac{\partial^{2} w^{*}}{\partial R^{* 2}}+\frac{1}{R^{*}} \cdot \frac{\partial w^{*}}{\partial R^{*}}+\frac{\partial^{2} w^{*}}{\partial z^{* 2}}\right)+ \\
\frac{1}{\operatorname{Re}} \cdot \frac{\partial H^{*}}{\partial R^{*}} \cdot\left(\frac{\partial u^{*}}{\partial z^{*}}+\frac{\partial w^{*}}{\partial R^{*}}\right)+\frac{2}{\operatorname{Re}} \cdot \frac{\partial H^{*}}{\partial z^{*}} \cdot \frac{\partial w^{*}}{\partial z^{*}}
\end{gathered}
$$

Using the above non-dimensional parameters, the energy equation is becoming:

$$
\frac{\partial^{2} T^{*}}{\partial R^{* 2}}+\frac{1}{R^{*}} \cdot \frac{\partial T^{*}}{\partial R^{*}}+\frac{\partial^{2} T^{*}}{\partial z^{* 2}}=0
$$

\section{Solution of the equations}

Resolving the system of eqns (4) to (8), it was found that the axial velocity $w^{*}$, the radial velocity $u^{*}$, the tangential velocity $v^{*}$ can be expressed in terms of the functions:

$$
\begin{gathered}
w^{*}=J_{0}(r b) \cdot e^{b \cdot z^{*}} \\
u^{*}=-J_{1}(r b) \cdot e^{b \cdot z^{*}} \\
v^{*}=-r
\end{gathered}
$$


where $J_{0}(r b)$ and $J_{1}(r b)$ are the Bessel functions of the first kind and $b=2,4056405$ defined in details in [11].

Pressure $P^{*}$, temperature $T^{*}$ and viscosity $H^{*}$ can be expressed in terms of the functions:

$$
\begin{gathered}
P^{*}=\frac{\xi}{2} \cdot\left[J_{0}^{2}(r b)+J_{1}^{2}(r b)\right] \cdot e^{2 \cdot b \cdot z^{*}} \\
\xi=\frac{2 \cdot b}{\operatorname{Re}}-1 \\
T^{*}=A+B \cdot J_{0}(r b) e^{b z} \\
H^{*}=J_{0}(b \cdot r) \cdot e^{b \cdot z}
\end{gathered}
$$

where the constants $A=\frac{1}{E c}, \quad B=-\frac{1}{E c}$

The above solutions (eqns 9 to 15 ) are implemented to the system of the nondimensional partial differential equations (4) to (8). The governing equations are satisfied, as shown below:

\subsection{Continuity equation}

Substituting the solutions for $u^{*}, w^{*}, v^{*}$ the continuity equation yields:

$$
\begin{gathered}
\frac{\partial\left(J_{1} \cdot e^{-b \cdot z^{*}} e^{k \cdot t}+\frac{B}{R^{*}}\right)}{\partial R^{*}}+\frac{J_{1} \cdot e^{-b \cdot z^{*}} e^{k \cdot t}+\frac{B}{R^{*}}}{R^{*}}+\frac{\partial\left(J_{0} \cdot e^{-b \cdot z^{*}} e^{k \cdot t}+A \cdot\left(1-R^{* 2}\right)\right)}{\partial z^{*}}= \\
\frac{J_{1}\left(b \cdot R^{*}\right) \cdot e^{-b \cdot z^{*}} e^{k \cdot t}}{R^{*}}+\frac{B}{R^{* 2}}+\left(b \cdot J_{0}\left(b \cdot R^{*}\right)-\frac{J_{1}\left(b \cdot R^{*}\right)}{R^{*}}\right) \cdot e^{-b \cdot z^{*}} e^{k \cdot t}+ \\
\left(-\frac{B}{R^{* 2}}\right)+(-b) \cdot J_{0}\left(b \cdot R^{*}\right) \cdot e^{-b \cdot z^{*}} e^{k \cdot t}=0
\end{gathered}
$$

meaning that the continuity equation is satisfied.

\subsection{R-momentum equation}

Introducing the expressions for the flow velocities $u^{*}, v^{*}, w^{*}$, in the $r$-momentum equation, one can see that these expressions satisfy the equation. 
The left hand side of the equation is:

$$
\begin{gathered}
\frac{\partial u^{*}}{\partial R^{*}}+w^{*} \cdot \frac{\partial u^{*}}{\partial z^{*}}-\frac{v^{* 2}}{R^{*}}=b \cdot\left(e^{b \cdot z^{*}}\right)^{2} \cdot J_{0}\left(b \cdot R^{*}\right) \cdot J_{1}\left(b \cdot R^{*}\right)- \\
\frac{\left(e^{b \cdot z^{*}}\right)^{2} \cdot\left(J_{1}\left(b \cdot R^{*}\right)\right)^{2}}{R^{*}}-b \cdot\left(e^{b \cdot z^{*}}\right)^{2} \cdot J_{0}\left(b \cdot R^{*}\right) \cdot J_{1}\left(b \cdot R^{*}\right)=-\frac{e^{2 \cdot b \cdot z^{*}} \cdot\left(J_{1}\left(b \cdot R^{*}\right)\right)^{2}}{R^{*}}-R^{*}
\end{gathered}
$$

The right hand-side terms of the equation are:

The term $\frac{\partial P^{*}}{\partial R^{*}}$ is becoming:

$$
\begin{aligned}
-\frac{\partial P^{*}}{\partial R^{*}}= & \frac{\left(\frac{2 b}{\operatorname{Re}}-1\right) \cdot e^{2 \cdot b \cdot z^{*}}}{2} \cdot \frac{\partial}{\partial R^{*}}\left[J_{1}^{2}\left(b \cdot R^{*}\right)+J_{0}^{2}\left(b \cdot R^{*}\right)\right] \\
& =\frac{2 \cdot b \cdot e^{2 \cdot b \cdot z^{*}} J_{1}^{2}\left(b \cdot R^{*}\right)}{\operatorname{Re} \cdot R^{*}}-\frac{e^{2 \cdot b \cdot z^{*}} J_{1}^{2}\left(b \cdot R^{*}\right)}{R^{*}}
\end{aligned}
$$

The terms:

$$
\begin{gathered}
\frac{H^{*}}{\operatorname{Re}} \cdot\left(\frac{\partial^{2} u^{*}}{\partial R^{* 2}}+\frac{1}{R^{*}} \cdot \frac{\partial u^{*}}{\partial R^{*}}-\frac{u^{*}}{R^{*}}+\frac{\partial^{2} u^{*}}{\partial z^{* 2}}\right)= \\
=\frac{H^{*}}{\operatorname{Re}} \cdot\left[b^{2} \cdot e^{b \cdot z^{*}} \cdot J_{1}\left(b \cdot R^{*}\right)+\frac{b \cdot e^{b \cdot z^{*}} \cdot J_{0}\left(b \cdot R^{*}\right)}{R^{*}}-\frac{2 \cdot e^{b \cdot z^{*}} J_{1}\left(b \cdot R^{*}\right)}{\left(R^{*}\right)^{2}}-\right. \\
\left.-\frac{b \cdot e^{b \cdot z^{*}} \cdot J_{0}\left(b \cdot R^{*}\right)}{R^{*}}+\frac{2 \cdot e^{b \cdot z^{*}} J_{1}\left(b \cdot R^{*}\right)}{\left(R^{*}\right)^{2}}-b^{2} \cdot e^{b \cdot z^{*}} \cdot J_{1}\left(b \cdot R^{*}\right)\right]=0
\end{gathered}
$$

The terms:

$$
\begin{gathered}
R^{*}+2 v^{*}+\frac{1}{\operatorname{Re}} \cdot \frac{\partial H^{*}}{\partial z} \cdot \frac{\partial u^{*}}{\partial z}+\frac{1}{\operatorname{Re}} \cdot \frac{\partial H^{*}}{\partial z} \cdot \frac{\partial w^{*}}{\partial R^{*}}+\frac{2}{\operatorname{Re}} \cdot \frac{\partial H^{*}}{\partial R^{*}} \cdot \frac{\partial u^{*}}{\partial R^{*}}= \\
=-R^{*}-\frac{2 b^{2} \cdot e^{2 b \cdot z^{*}} \cdot J_{0}\left(b \cdot R^{*}\right) \cdot J_{1}\left(b \cdot R^{*}\right)}{\operatorname{Re}}+ \\
\frac{2}{\operatorname{Re}} \cdot\left(2 b^{2} \cdot e^{2 b \cdot z^{*}} \cdot J_{0}\left(b \cdot R^{*}\right) \cdot J_{1}\left(b \cdot R^{*}\right)-\frac{2 b \cdot e^{2 b \cdot z^{*}} \cdot J_{1}^{2}\left(b \cdot R^{*}\right)}{R^{*}}\right)= \\
=-R^{*}-\frac{2 b \cdot e^{2 b \cdot z^{*}} \cdot J_{1}^{2}\left(b \cdot R^{*}\right)}{\operatorname{Re} \cdot R^{*}} \equiv \frac{\partial P^{*}}{\partial R^{*}}
\end{gathered}
$$

Thus, we see that the $r$-momentum equation satisfies the proposed solution. 


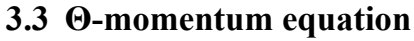

Introducing the expressions for the flow velocities $u^{*}, v^{*}, w^{*}$, in the $\theta$-momentum equation, one can see that these expressions satisfy the equation.

The left hand side of the equation is:

$$
\begin{gathered}
u^{*} \cdot \frac{\partial v^{*}}{\partial R^{*}}+w^{*} \cdot \frac{\partial v^{*}}{\partial z^{*}}+\frac{u^{*} \cdot v^{*}}{R^{*}}=\left(-J_{1}\left(b \cdot R^{*}\right) \cdot e^{b \cdot z^{*}}\right) \cdot \frac{\partial\left(-R^{*}\right)}{\partial R^{*}}+ \\
\left(J_{0}\left(b \cdot R^{*}\right) \cdot e^{b \cdot z^{*}}\right) \cdot \frac{\partial\left(-R^{*}\right)}{\partial z^{*}}+\frac{\left(-J_{1}\left(b \cdot R^{*}\right) \cdot e^{b \cdot z^{*}}\right) \cdot\left(-R^{*}\right)}{R^{*}}= \\
-J_{1}\left(b \cdot R^{*}\right) \cdot e^{b \cdot z^{*}}+J_{1}\left(b \cdot R^{*}\right) \cdot e^{b \cdot z^{*}}=0
\end{gathered}
$$

The right-hand side of the $\theta$-momentum equation is:

The term

$$
\begin{gathered}
\frac{1}{\operatorname{Re}} \cdot \frac{\partial \mathrm{H}^{*}}{\partial z^{*}} \cdot \frac{\partial v^{*}}{\partial z^{*}}=\frac{1}{\operatorname{Re}} \cdot\left(b \cdot J_{0}\left(b \cdot R^{*}\right) \cdot e^{b \cdot z^{*}}\right) \cdot \frac{\partial\left(-R^{*}\right)}{\partial z^{*}}= \\
\frac{1}{\operatorname{Re}} \cdot\left(b \cdot J_{0}\left(b \cdot R^{*}\right) \cdot e^{b \cdot z^{*}}\right) \cdot 0=0
\end{gathered}
$$

The term

$$
\begin{aligned}
\frac{1}{\operatorname{Re}} \cdot \frac{\partial \mathrm{H}^{*}}{\partial R^{*}} \cdot\left(\frac{\partial v^{*}}{\partial R^{*}}\right. & \left.-\frac{v^{*}}{R^{*}}\right)= \\
& \frac{1}{\operatorname{Re}} \cdot\left(-b \cdot J_{1}\left(b \cdot R^{*}\right) \cdot e^{b \cdot z^{*}}\right) \cdot\left[\frac{\partial\left(-R^{*}\right)}{\partial R^{*}}-\frac{\left(-R^{*}\right)}{R^{*}}\right]=0
\end{aligned}
$$

The term

$$
\begin{aligned}
& \frac{\omega^{2} \cdot L \cdot H}{\operatorname{Re}}\left[\frac{\partial}{\partial R^{*}}\left(\frac{\partial v^{*}}{\partial R^{*}}\right)+\frac{1}{R^{*}} \cdot \frac{\partial v^{*}}{\partial R^{*}}-\frac{v^{*}}{R^{* 2}}+\frac{\partial}{\partial z^{*}}\left(\frac{\partial v^{*}}{\partial z^{*}}\right)\right]= \\
& \frac{J_{0}\left(b \cdot R^{*}\right) \cdot e^{b \cdot z^{*}}}{\operatorname{Re}} \cdot\left[\frac{\partial}{\partial R^{*}}(-1)+\frac{1}{R^{*}} \cdot(-1)+\frac{R^{*}}{R^{* 2}}+0\right]= \\
& \frac{J_{0}\left(b \cdot R^{*}\right) \cdot e^{b \cdot z^{*}}}{\operatorname{Re}} \cdot\left(-\frac{1}{R^{*}}+\frac{1}{R^{*}}\right)=0
\end{aligned}
$$

Hence the proposed solution satisfies the $\theta$-momentum equation. 


\subsection{Z-momentum equation}

Introducing the expressions for the flow velocities $u^{*}, v^{*}, w^{*}$, in the $\mathrm{z}$-momentum equation, one can see that these expressions satisfy the equation.

The left hand side of the equation is:

$$
\begin{gathered}
u^{*} \cdot \frac{\partial w^{*}}{\partial R^{*}}+w^{*} \cdot \frac{\partial w^{*}}{\partial z^{*}}= \\
=\left(-J_{1}\left(b \cdot R^{*}\right) \cdot e^{b \cdot z^{*}}\right) \cdot\left(-b \cdot e^{b \cdot z^{*}} \cdot J_{1}\left(b \cdot R^{*}\right)\right)+\left(J_{0}\left(b \cdot R^{*}\right) \cdot e^{b \cdot z^{*}}\right) \cdot \\
\frac{\partial\left(J_{0}\left(b \cdot R^{*}\right) \cdot e^{b \cdot z^{*}}\right)}{\partial z^{*}}=b \cdot e^{2 \cdot b \cdot z^{*}} \cdot\left[J_{1}^{2}\left(b \cdot R^{*}\right)+J_{0}^{2}\left(b \cdot R^{*}\right)\right]
\end{gathered}
$$

The term $-\frac{\partial P^{*}}{\partial z^{*}}$ can be written:

$$
\begin{aligned}
& -\frac{\partial P^{*}}{\partial z^{*}}=-\frac{\partial\left[\frac{\xi}{2} \cdot\left(J_{1}^{2}\left(b \cdot R^{*}\right)+J_{0}^{2}\left(b \cdot R^{*}\right)\right)\right]}{\partial z^{*}}= \\
& -\frac{\xi}{2} \cdot\left[J_{1}^{2}\left(b \cdot R^{*}\right)+J_{0}^{2}\left(b \cdot R^{*}\right)\right] \cdot \frac{\partial\left(e^{2 \cdot b \cdot z^{*}}\right)}{\partial z^{*}}= \\
& -\xi \cdot\left[J_{1}^{2}\left(b \cdot R^{*}\right)+J_{0}^{2}\left(b \cdot R^{*}\right)\right] \cdot b \cdot e^{2 \cdot b \cdot z^{*}}= \\
& b \cdot e^{2 \cdot b \cdot z^{*}} \cdot\left(\frac{2 \cdot b}{\operatorname{Re}}-1\right) \cdot\left[J_{1}^{2}\left(b \cdot R^{*}\right)+J_{0}^{2}\left(b \cdot R^{*}\right)\right]
\end{aligned}
$$

The term

$$
\begin{gathered}
\frac{H^{*}}{\operatorname{Re}} \cdot\left(\frac{\partial^{2} w^{*}}{\partial R^{* 2}}+\frac{1}{R^{*}} \cdot \frac{\partial w^{*}}{\partial R^{*}}+\frac{\partial^{2} w^{*}}{\partial z^{* 2}}\right)= \\
\frac{J_{0}\left(b \cdot R^{*}\right) \cdot e^{b \cdot z^{*}}}{\operatorname{Re}} \cdot\left[\begin{array}{l}
-b^{2} \cdot e^{b \cdot z^{*}} \cdot J_{0}\left(b \cdot R^{*}\right)+b \cdot e^{b \cdot z^{*}} \cdot \frac{J_{1}\left(b \cdot R^{*}\right)}{R^{*}}- \\
b \cdot e^{b \cdot z^{*}} \cdot \frac{J_{1}\left(b \cdot R^{*}\right)}{R^{*}}+b^{2} \cdot e^{b \cdot z^{*}} \cdot J_{0}\left(b \cdot R^{*}\right)
\end{array}\right]=0
\end{gathered}
$$


The term

$$
\frac{1}{\operatorname{Re}} \cdot \frac{\partial H^{*}}{\partial R^{*}} \cdot\left(\frac{\partial u^{*}}{\partial z^{*}}+\frac{\partial w^{*}}{\partial R^{*}}\right)=\frac{2 \cdot b^{2} \cdot e^{2 \cdot b \cdot z^{*}} \cdot J_{1}^{2}\left(b \cdot R^{*}\right)}{\operatorname{Re}}
$$

The term

$$
\begin{aligned}
& \frac{2}{\operatorname{Re}} \cdot \frac{\partial H^{*}}{\partial z^{*}} \cdot \frac{\partial w^{*}}{\partial z^{*}}=\frac{2}{\operatorname{Re}} \cdot {\left[\left(b \cdot e^{b \cdot z^{*}} \cdot J_{0}\left(b \cdot R^{*}\right)\right) \cdot\left(b \cdot e^{b \cdot z^{*}} \cdot J_{0}\left(b \cdot R^{*}\right)\right)\right]=} \\
& \frac{2 \cdot b^{2} \cdot e^{2 \cdot b \cdot z^{*}} \cdot J_{0}^{2}\left(b \cdot R^{*}\right)}{\operatorname{Re}}
\end{aligned}
$$

Substituting the above expressions to the non-dimensional form of the $\mathrm{z}$ momentum equation, we obtain:

$$
\begin{gathered}
b \cdot e^{2 \cdot b \cdot z^{*}} \cdot\left[J_{1}^{2}\left(b \cdot R^{*}\right)+J_{0}^{2}\left(b \cdot R^{*}\right)\right]= \\
-b \cdot e^{2 \cdot b \cdot z^{*}} \cdot\left(\frac{2 \cdot b}{\operatorname{Re}}-1\right) \cdot\left[J_{1}^{2}\left(b \cdot R^{*}\right)+J_{0}^{2}\left(b \cdot R^{*}\right)\right]+ \\
\frac{2 \cdot b^{2} \cdot e^{2 \cdot b \cdot z^{*}} \cdot J_{1}^{2}\left(b \cdot R^{*}\right)}{\operatorname{Re}}+\frac{2 \cdot b^{2} \cdot e^{2 \cdot b \cdot z^{*}} \cdot J_{0}^{2}\left(b \cdot R^{*}\right)}{\operatorname{Re}}
\end{gathered}
$$

which equals the left hand side, meaning that proposed solution for the temperature satisfies the $\mathrm{z}$-momentum equation.

\subsection{Energy equation}

$$
\frac{\partial^{2} T^{*}}{\partial R^{* 2}}+\frac{1}{R^{*}} \cdot \frac{\partial T^{*}}{\partial R^{*}}+\frac{\partial^{2} T^{*}}{\partial z^{* 2}}=0
$$

The term

$$
\frac{\partial T^{*}}{\partial R^{*}}=\frac{\partial\left(A+B \cdot J_{o}\left(b \cdot R^{*}\right) \cdot e^{b \cdot z^{*}}\right)}{\partial R^{*}}=-B \cdot b \cdot e^{b \cdot z^{*}} \cdot J_{1}\left(b \cdot R^{*}\right)
$$

The term

$$
\frac{\partial T^{*}}{\partial z^{*}}=\frac{\partial\left(A+B \cdot J_{o}\left(b \cdot R^{*}\right) \cdot e^{b \cdot z^{*}}\right)}{\partial z^{*}}=B \cdot b \cdot e^{b \cdot z^{*}} \cdot J_{0}\left(b \cdot R^{*}\right)
$$


The derivative

$$
\begin{gathered}
\frac{\partial\left(-B \cdot b \cdot e^{b \cdot z^{*}} \cdot J_{1}\left(b \cdot R^{*}\right)\right)}{\partial R^{*}}= \\
-b^{2} \cdot B \cdot e^{b \cdot z^{*}} \cdot J_{0}\left(b \cdot R^{*}\right)+\frac{B \cdot b \cdot e^{b \cdot z^{*}} \cdot J_{1}\left(b \cdot R^{*}\right)}{R^{*}}
\end{gathered}
$$

The derivative

$$
\frac{\partial\left(B \cdot b \cdot e^{b \cdot z^{*}} \cdot J_{0}\left(b \cdot R^{*}\right)\right)}{\partial z^{*}}=b^{2} \cdot B \cdot e^{b \cdot z^{*}} \cdot J_{0}\left(b \cdot R^{*}\right)
$$

Thus the energy equation is:

$$
\begin{gathered}
-b^{2} \cdot B \cdot e^{b \cdot z^{*}} \cdot J_{0}\left(b \cdot R^{*}\right)+\frac{B \cdot b \cdot e^{b \cdot z^{*}} \cdot J_{1}\left(b \cdot R^{*}\right)}{R^{*}}-\frac{B \cdot b \cdot e^{b \cdot z^{*}} \cdot J_{1}\left(b \cdot R^{*}\right)}{R^{*}} \\
+b^{2} \cdot B \cdot e^{b \cdot z^{*}} \cdot J_{0}\left(b \cdot R^{*}\right)=0
\end{gathered}
$$

which means that the proposed solution for the temperature satisfies the energy equation.

The distributions of axial and radial velocity components in terms of the nondimensional radius $R^{*}$ for different axial positions $z^{*}$, are shown in figure 1 . One can see that the axial velocity distribution (figure 1a) respects the condition that its value is zero at $R^{*}=1$ for all axial positions $z^{*}$. Similarly, the radial velocity distribution (figure $1 \mathrm{~b}$ ) respects the condition that for all axial positions $z^{*}$ its values is zero at $R *=0$.

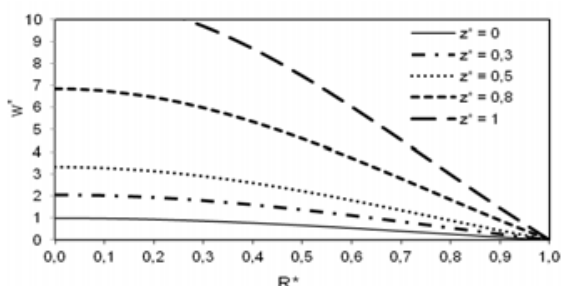

(a)

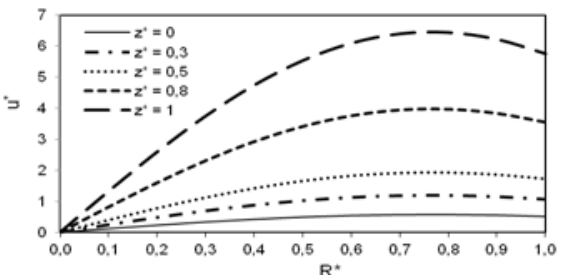

(b)

Figure 1: (a) Axial and (b) radial velocity distribution in terms of $\mathrm{R}^{*}$.

The temperature and the pressure distributions in terms of the nondimensional radius $R^{*}$ for different axial positions, are shown in figure 2. From the temperature distribution, it can be seen that at the outer radial boundary corresponding at $R^{*}=1$ all the curves converge to the same value of temperature. 
The fact that from figure $2 \mathrm{a}$ we can observe variations of temperature in terms of the radius and in terms of axial coordinate, reveals a three-dimensional field of the distribution.

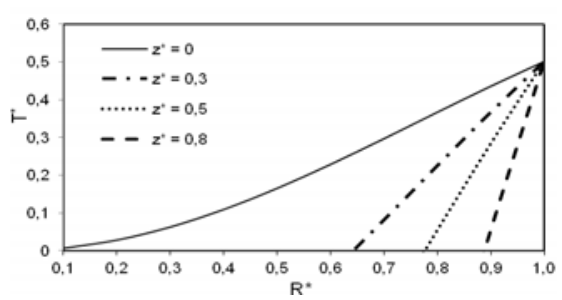

(a)

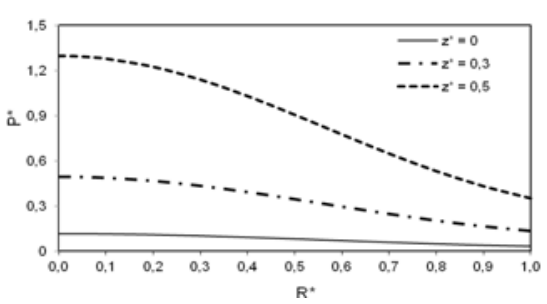

(b)

Figure 2: $\quad$ (a) Temperature and (b) pressure distribution in terms of $\mathrm{R}^{*}$.

Similar observations occur for the case of the pressure field. The pressure variations at the inlet corresponding at $z^{*}=0$ are negligible, whereas the variations are more intense as the axial distance $z *$ increases.

\section{Conclusions}

In this article, an original work has presented an exact solution of the Navier-Stokes equations in cylindrical coordinates for incompressible, laminar, axisymmetric, viscous flows with constant and variable viscosity. The fluid viscosity was assumed to be a function of temperature, as literature survey indicated for flows in rotating machinery [10]. It was proven that the solution field consisting of the Bessel functions of the first kind and of exponential functions satisfies the continuity equation, the equations of motion and the energy equation. It was shown that the present method based on Bessel functions can be used to provide reference solutions for numerical and empirical methods for flow field predictions in rotating systems involving fluids of variable viscosity.

\section{References}

[1] Drazin P.G. and Riley N., The Navier-Stokes equations: a classification of flows and exact solutions, London Mathematical Society Lecture Note Series, Cambridge University Press, 2007.

[2] Oldroyd J.G., On the formulation of rheological equations of state, Proc. $R$. Soc. London A200, pp. 523-541, 1950.

[3] Fetecau C., Analytical solutions for non-Newtonian fluid flows in pipe-like domains, Int. J. of Non-linear Mechanics, 39, pp. 225-231, 2004.

[4] Martin M.H., The flow of a viscous fluid, Arch. Rat. Mech. Anal. 41, pp. 266-286, 1971. 
[5] Naeem R.K. and Nadeem S.A., Study of plane steady flows of an incompressible fluid of variable viscosity using Martins method, International Journal of Applied Mechanics and Engineering, 1(3), pp. 397-433, 1996.

[6] Naeem R.K., A class of exact solutions of the Navier-Stokes equations for incompressible fluid of variable viscosity for defined vorticity distribution, International Journal of Applied Mathematics and Mechanics 7(4), pp. 97$118,2011$.

[7] Mahanti N.C. and Gaur P., Effects of Varying Viscosity and Thermal Conductivity on Steady Free Convective Flow and Heat Transfer along an Isothermal Vertical Plate in the Presence of Heat Sink, Journal of Applied Fluid Mechanics, 2(1), pp. 23-28, 2009.

[8] Makinde O.D. and Maserumule R.L., Thermal criticality and entropy analysis for a variable viscosity Couette flow, Psys. Scr., 78, pp. 1-6, 2008.

[9] Bird R.B., Stewart W.E. Lightfoot E.N., Transport Phenomena, $2^{\text {nd }}$ edition, John Wiley and Sons, Inc., 2002.

[10] Schilling R., Siegle H. and Stoffel B, Strömung und Verluste in drei wichigen Elementen radialer Kreiselpumpen. Eine Literaturübersicht, Universität Karlsruhe (TH), Nr. 16, 1974.

[11] Kreuszig, E., Advanced Engineering Mathematics, $11^{\text {th }}$ edition, John Wiley and Sons Inc., pp. 187-191, 2011. 